\title{
Finding the Smallest Eigenvalue by the Inverse Monte Carlo Method with Refinement*
}

\author{
Vassil Alexandrov ${ }^{1}$ and Aneta Karaivanova ${ }^{2}$ \\ 1 School of Systems Engineering, University of Reading, Reading RG6 6AY, UK \\ v.n.alexandrov@rdg.ac.uk \\ http://www.cs.reading.ac.uk/people/V.Alexandrov.htm \\ 2 IPP - BAS, Acad. G. Bonchev St., Bl.25A, 1113 Sofia, Bulgaria \\ anet@parallel.bas.bg \\ http://parallel.bas.bg/ anet/
}

\begin{abstract}
Finding the smallest eigenvalue of a given square matrix $A$ of order $n$ is computationally very intensive problem. The most popular method for this problem is the Inverse Power Method which uses $L U$ decomposition and forward and backward solving of the factored system at every iteration step. An alternative to this method is the Resolvent Monte Carlo method which uses representation of the resolvent matrix $[I-q A]^{-m}$ as a series and then performs Monte Carlo iterations (random walks) on the elements of the matrix. This leads to great savings in computations, but the method has many restrictions and a very slow convergence.

In this paper we propose a method that includes fast Monte Carlo procedure for finding the inverse matrix, refinement procedure to improve approximation of the inverse if necessary, and Monte Carlo power iterations to compute the smallest eigenvalue. We provide not only theoretical estimations about accuracy and convergence but also results from numerical tests performed on a number of test matrices.
\end{abstract}

Keywords: Monte Carlo methods, eigenvalues, Markov chains, parallel computing, parallel efficiency.

\section{Introduction}

Let $A$ be a real symmetric matrix. Consider the problem of evaluating the eigenvalues of $A$, i.e. the values of $\lambda$ for which

$$
A u=\lambda u
$$

holds. Suppose, the $n$ eigenvalues of $A$ are ordered as follows $\left|\lambda_{1}\right|>\left|\lambda_{2}\right| \geq \ldots \geq$ $\left|\lambda_{n-1}\right|>\left|\lambda_{n}\right|$.

\footnotetext{
* Supported by the Ministry of Education and Science of Bulgaria under Grant No. I1405/04.
} 
It is known that the problem of calculating the smallest eigenvalue of $A$ is more difficult from numerical point of view than the problem of evaluating the largest eigenvalue. Nevertheless, for many applications in physics and engineering it is important to estimate the smallest one, because it usually defines the most stable state of the system which is described by the considered matrix.

\section{$2 \quad$ Background}

\subsection{Inverse Power Method}

One of the most popular methods for finding extremal eigenvalues is the power method $([6], 8])$ which for a given matrix $A$ is defined by the iteration

$$
x^{\text {new }}=A x^{\text {old }} \text {. }
$$

Except for special starting points, the iterations converge to an eigenvector corresponding to the eigenvalue of $A$ with largest magnitude (dominant eigenvalue). The least squares solution $\mu$ to the overdetermined linear system

$$
\mu x_{k}=x_{k+1}
$$

is an estimate for $\lambda_{1}$ which is called the Raleigh quotient.

$$
\mu=\frac{x_{k}^{T} x_{k+1}}{x_{k}^{T} x_{k}} .
$$

Suppose that we want to compute the eigenvector corresponding to the eigenvalue of $A$ of smallest magnitude. Letting $\left(\lambda_{1}, e_{1}\right)$ through $\left(\lambda_{n}, e_{n}\right)$ denote the eigenpairs of $A$, the corresponding eigenpairs of $C=A^{-1}$ are $\left(1 / \lambda_{1}, e_{1}\right)$ through $\left(1 / \lambda_{n}, e_{n}\right)$. If $\lambda_{n}$ is the eigenvalue of $A$ of smallest magnitude, then $1 / \lambda_{n}$ is $C^{\prime}$ s eigenvalue of largest magnitude and the power iteration $x^{\text {new }}=A^{-1} x^{\text {old }}$ converges to the vector $e_{n}$ corresponding to the eigenvalue $1 / \lambda_{n}$ of $C=A^{-1}$. When implementing the inverse power method, instead of computing the inverse matrix $A^{-1}$ we multiply by $A$ to express the iteration $x^{\text {new }}=A^{-1} x^{\text {old }}$ in the form $A x^{\text {new }}=x^{\text {old }}$. Replacing $A$ by its $L U$ factorization yields

$$
(L U) x^{\text {new }}=x^{\text {old }} .
$$

In each iteration of the inverse power method, the new $x$ is obtained from the old $x$ by forward and back solving the factored system (2). This scheme is computationally more efficient. With $k$ iterations, the number of arithmetic operations is $O\left(k n^{2}\right)$ for Power method and $O\left(n^{3}+k n^{2}\right)$ for Inverse Power Method. 


\subsection{Resolvent Monte Carlo Method}

Given a matrix $A$, consider an algorithm based on Monte Carlo iterations by the resolvent matrix $R_{q}=[I-q A]^{-1} \in \mathbb{R}^{n \times n}$. The following representation

$$
[I-q A]^{-m}=\sum_{i=0}^{\infty} q^{i} C_{m+i-1}^{i} A^{i},|q \lambda|<1
$$

is valid because of behaviors of binomial expansion and the spectral theory of linear operators. Let remind that the eigenvalues of the matrices $R_{q}$ and $A$ are connected by the equality $\mu=\frac{1}{1-q \lambda}$, and the eigenvectors coincide. The following expression (see [4])

$$
\mu^{(m)}=\frac{\left([I-q A]^{-m} f, h\right)}{\left([I-q A]^{-(m-1)} f, h\right)} \rightarrow_{m \rightarrow \infty} \mu=\frac{1}{1-q \lambda}, \quad f \in \mathbb{R}^{n}, h \in \mathbb{R}^{n},
$$

is valid ( $\mathrm{f}$ and $\mathrm{h}$ are vectors in $\mathbb{R}^{n}$ ). For negative values of $q$, the largest eigenvalue $\mu_{\max }$ of $R_{q}$ corresponds to the smallest eigenvalue $\lambda_{\min }$ of the matrix $A$.

Moreover, if $\left|\lambda_{\max }^{\prime}<1\right|$ where $\lambda_{\max }^{\prime}$ is the largest eigenvalue of the matrix $A^{\prime}=\left\{\left|a_{i j}\right|\right\}_{i, j=1}^{n}$, the following statement holds, 4], 2]:

$$
\left([I-q A]^{-m} f, h\right)=E\left\{\sum_{i=0}^{\infty} q^{i} C_{m+i-1}^{i}\left(A^{i} f, h\right)\right\} .
$$

Now we can construct Monte Carlo method. Define Markov chain $k_{0} \rightarrow k_{1} \rightarrow$ $\ldots \rightarrow k_{i}\left(1 \leq k_{j} \leq n\right.$ are natural numbers $)$ with initial and transition densities, $\operatorname{Pr}\left(k_{0}=\alpha\right)=p_{\alpha}, \operatorname{Pr}\left(k_{j}=\beta \mid k_{j-1}=\alpha\right)=p_{\alpha \beta},($ see [4], [2]). Now define the random variables $W_{j}$ using the following recursion formula:

$$
W_{0}=\frac{h_{k_{0}}}{p_{k_{0}}}, \quad W_{j}=W_{j-1} \frac{a_{k_{j-1} k_{j}}}{p_{k_{j-1} k_{j}}}, j=1, \ldots, i .
$$

Than it can be proven that (see [4, [2]):

$$
\begin{gathered}
\lambda_{\min } \approx \frac{1}{q}\left(1-\frac{1}{\mu^{(m)}}\right)=\frac{\left(A[I-q A]^{-m} f, h\right)}{\left([I-q A]^{-m} f, h\right)}= \\
\frac{E \sum_{i=1}^{\infty} q^{i-1} C_{i+m-2}^{i-1} W_{i} f_{x_{i}}}{E \sum_{i=0}^{\infty} q^{i} C_{i+m-1}^{i} W_{i} f_{x_{i}}}=\frac{E \sum_{i=0}^{l} q^{i} C_{i+m-1}^{i} W_{i+1} f_{x_{i}}}{E \sum_{i=0}^{l} q^{i} C_{i+m-1}^{i} W_{i} f_{x_{i}}}
\end{gathered}
$$

where $W_{0}=\frac{h_{k_{0}}}{p_{k_{0}}}$ and $W_{i}$ are defined by (15). The coefficients $C_{i+m}^{n}$ are calculated using the presentation $C_{i+m}^{i}=C_{i+m-1}^{i}+C_{i+m-1}^{i-1}$.

This method has strong requirements about matrices for which it can be applied. The systematic error consists of two parts: 
- an error from the Power method applied on the resolvent matrix $[I-q A]^{-1}$ which determines the value of the parameter $m$ in the following way: The rate of convergence is

$$
O\left(\left(\frac{1+|q| \lambda_{n}}{1+|q| \lambda_{n-1}}\right)^{m}\right) .
$$

The parameter $m$ has to be chosen such that $\left(\frac{\mu_{2}}{\mu_{1}}\right)^{m}<\varepsilon$.

- an error which comes from the series expansion of the resolvent matrix it determines the value of the parameter $l$ (length of each random walk). Considering the presentation

$$
\left([I-q A]^{-m} f, g\right) \approx \sum_{i=0}^{l} q^{i} C_{m+i-1}^{i}\left(A^{i} f, g\right)=\sum_{i=1}^{l} u_{i}
$$

the parameter $l$ has to be chosen such that $\left|u_{l+1}\right|<\varepsilon_{2}$.

- rounding errors - to maintain them at a low level the condition $\left(\max _{1 \leq i \leq l}\left|u_{i}\right|\right) \varepsilon$ $<\alpha \mu$ must be satisfied, where $\alpha<1$ represents the requested precision and $\varepsilon$ is the machine precision parameter.

Unfortunately, the parameter $l$ can not be relatively large because the binomial coefficients $C_{m+l-1}^{l}$ grow exponentially with $l$. This is serious restriction for using the resolvent Monte Carlo.

The Resolvent Monte Carlo method computes the smallest eigenvalue with only $O(l N)$ operations, where $l$ is the average length of the Markov chains and $N$ is the number of walks. But this method has a lot of restrictions, and, also, gives very rough approximation.

\section{$3 \quad$ Inverse Monte Carlo with Refinement}

Here we propose a method that includes fast Monte Carlo scheme for matrix inversion, refinement of the inverse matrix (if necessary) and Monte Carlo power iterations for computing the largest eigenvalue of the inverse (the smallest eigenvalue of the given matrix).

\subsection{Monte Carlo for Computing the Inverse Matrix}

To find the inverse $A^{-1}=C=\left\{c_{r r^{\prime}}\right\}_{r, r^{\prime}=1}^{n}$ of some matrix $A$, we must first compute the elements of matrix $M=I-A$, where $I$ is the identity matrix. Clearly, the inverse matrix is given by

$$
C=\sum_{i=0}^{\infty} M^{i}
$$

which converges if $\|M\|<1$ (sufficient condition). 
To estimate the element $c_{r r^{\prime}}$ of the inverse matrix $C$, we define Markov chain $s_{0} \rightarrow s_{1} \rightarrow \cdots \rightarrow s_{k}$, where the $s_{i}, i=1,2, \cdots, k$, belongs to the state space $S=\{1,2, \cdots, n\}$. Then for $\alpha, \beta \in S, p_{0}(\alpha)=p\left(s_{0}=\alpha\right)$ is the probability that the Markov chain starts at state $\alpha$ and $p\left(s_{j+1}=\beta \mid s_{j}=\alpha\right)=p_{\alpha \beta}$ is the transition probability from state $\alpha$ to state $\beta$. The set of all probabilities $p_{\alpha \beta}$ defines a transition probability matrix $P=\left\{p_{\alpha \beta}\right\}_{\alpha, \beta=1}^{n}$.

We then can use the following Monte Carlo method (see, for example, 11], [3]) for calculating elements of the inverse matrix $C$ :

$$
c_{r r^{\prime}} \approx \frac{1}{N} \sum_{s=1}^{N}\left[\sum_{\left(j \mid s_{j}=r^{\prime}\right)} W_{j}\right],
$$

where $\left(j \mid s_{j}=r^{\prime}\right)$ means that only

$$
W_{j}=\frac{M_{r s_{1}} M_{s_{1} s_{2}} \ldots M_{s_{j-1} s_{j}}}{p_{r s_{1}} p_{s_{1} s_{2}} \ldots p_{s_{j-1} p_{j}}}
$$

for which $s_{j}=r^{\prime}$ are included in the sum (8).

Since $W_{j}$ is included only into the corresponding sum for $r^{\prime}=1,2, \ldots, n$, then the same set of $N$ chains can be used to compute a single row of the inverse matrix, which is one of the inherent properties of MC making them suitable for parallelization.

Furthermore, if necessary we transform matrix $A$ to matrix $D$ where $D$ is diagonally dominant matrix, we find its inverse and after that apply a filter procedure in order to find $A^{-1}[5]$.

The probable error of the method, is defined as $r_{N}=0.6745 \sqrt{D \theta / N}$, where $P\left\{|\bar{\theta}-E(\theta)|<r_{N}\right\} \approx 1 / 2 \approx P\left\{|\bar{\theta}-E(\theta)|>r_{N}\right\}$, if we have $N$ independent realizations of random variable (r.v.) $\theta$ with mathematical expectation $E \theta$ and average $\bar{\theta}[1]$.

\subsection{Refinement}

Given a square nonsingular matrix $A$, Monte Carlo gives fast but very rough estimation of the elements of the inverse matrix $C_{0}$. Under the condition $\left\|R_{0}\right\| \leq$ $\delta<1$ where $R_{0}=I-A C_{0}$, the elements of $A^{-1}$ may be computed to whatever degree of accuracy is convenient using the iterative process: $C_{m}=C_{m-1}(I+$ $\left.R_{m-1}\right), R_{m}=I-A C_{m}$ for $m=1,2, \ldots$ It can be shown that

$$
\left\|C_{m}-A^{-1}\right\| \leq\left\|C_{0}\right\| \frac{\delta^{2^{m}}}{1-\delta} .
$$

It follows that once we have an initial approximation $C_{0}$ of the inverse such that $\left\|I-A C_{0}\right\| \leq k<1$ we can use the above iterative scheme and than the number of correct digits increases in geometric progression.

If we have inverted matrix $D$ then we need to apply the filter procedure to obtain the $A^{-1}$ using the formula

$$
A_{k}^{-1}=A_{k+1}^{-1}+\frac{A_{k+1}^{-1} S_{i+1} A_{k+1}^{-1}}{1-\operatorname{trace}\left(A_{k+1}^{-1} S_{i+1}\right)}, i=k-1, k-2, \ldots, 1,0 .
$$




\subsection{Evaluation of the Smallest Eigenvalue}

Once we have the matrix $C=A^{-1}$ we apply the Monte Carlo power iterations to find its largest eigenvalue performing random walks on the elements of the matrix $C$ :

$$
\lambda_{\max }(C) \approx \frac{E\left\{W_{m} f_{k_{i}}\right\}}{E\left\{W_{m-1} f_{k_{i-1}}\right\}} .
$$

Here $W_{j}=\frac{C_{k_{0} k_{1}} C_{k_{1} k_{2} \ldots C_{k_{j-1} k_{j}}}}{p_{k_{0} k_{1}} p_{k_{1} k_{2} \ldots p_{k_{j-1}} p_{j}}}$, and $m$ is sufficiently large to ensure the convergence of the power method which is $O\left(\frac{\lambda_{n}}{\lambda_{n-1}}\right)^{m}$.

The computational cost of power Monte Carlo is $m N$, where $m$ is determined by the convergence of the power iterations and $N$ is the number of walks.

\subsection{Restrictions of the Method and How to Avoid Them}

Suppose that $\lambda_{n}<\lambda_{n-1}$; in this case the power method is convergent. The only possible restriction in the above scheme is the requirement $\|M\|<1$ for the convergence of the Monte Carlo inversion. This can easily be avoided using the presented bellow algorithm for inverting general type matrices using Monte Carlo:

Algorithm: Finding $A^{-1}$.

1. Initial data: Input matrix $A$, parameters $\gamma$ and $\epsilon$.

2. Preprocessing:

2.1 Split $A=D-(D-A)$, where $D$ is a diagonally dominant matrix.

2.2 Set $D=B-B_{1}$ where B is a diagonal matrix $b_{i i}=d_{i i} i=1,2, \ldots, n$.

2.3 Compute the matrix $T=B^{-1} B_{1}$.

2.4 Compute $\|T\|$, the Number of Markov Chains $N=\left(\frac{0.6745}{\epsilon} \cdot \frac{1}{(1-|| T \|}\right)^{2}$.

3. For $\mathrm{i}=1$ to $\mathrm{n}$;

3.1 For $\mathrm{j}=1$ to $\mathrm{j}=\mathrm{N}$;

\section{Markov Chain Monte Carlo Computation:}

3.1.1 Set $t_{k}=0$ (stopping rule), $W_{0}=1, S U M[i]=0$ and Point $=i$.

3.1.2 Generate an uniformly distributed random number nextpoint.

3.1 .3 If $T[$ point $][$ nextpoint $] !=0$.

\section{LOOP}

3.1.3.1 Compute $W_{j}=W_{j-1} \frac{T[\text { point }][\text { nextpoint }]}{P[\text { point }][\text { nextpoint }]}$.

3.1.3.2 Set Point $=$ nextpoint and $S U M[i]=S U M[i]+W_{j}$.

3.1.3.3 If $\left|W_{j}\right|<\gamma, t_{k}=t_{k}+1$

3.1.3.4 If $t_{k} \geq n$, end LOOP.

\subsubsection{End If}

3.1.5 Else go to step 3.1.2.

\subsection{End of loop j.}

3.3 Compute the average of results.

4. End of loop i. 
5. Obtain The matrix $V=(I-T)^{-1}$.

6. Therefore $D^{-1}=V B^{-1}$.

7. Compute the $\mathrm{MC}$ inversion $D^{-1}=B(I-T)^{-1}$.

8. Set $D_{0}=D^{-1}$ (approximate inversion) and $R_{0}=I-D D_{0}$.

9. use filter procedure $R_{i}=I-D D_{i}, D_{i}=D_{i-1}\left(I+R_{i-1}\right), i=1,2, \ldots, m$, where $m \leq k$.

10. Consider the accurate inversion of $\mathbf{D}$ by step 9 given by $D_{0}=D_{k}$.

11. Compute $S=D-A$ where $S$ can be any matrix with all non-zero elements in diagonal and all of its off-diagonal elements are zero.

12. Main function for obtaining the inversion of A based on $D^{-1}$ step 9:

12.1 Compute the matrices $S_{i}, i=1,2, \ldots, k$, where each $S_{i}$ has just one element of matrix $S$.

12.2 Set $A_{0}=D_{0}$ and $A_{k}=A+S$

12.3 Apply $A_{k}^{-1}=A_{k+1}^{-1}+\frac{A_{k+1}^{-1} S_{i+1} A_{k+1}^{-1}}{1-\operatorname{trace}\left(A_{k+1}^{-1} S_{i+1}\right)}, i=k-1, k-2, \ldots, 1,0$.

13. Printthe inversion of matrix A.

14. End of algorithm.

\section{Parallel Implementation and Numerical Tests}

The method presented in this paper consists of 3 procedures:

- Monte Carlo inversion

- Refinement

- Power Monte Carlo iterations

We study the convergence and the parallel behaviour of each of the above procedures separately in order to have the overall picture.

It is well known that Monte Carlo algorithms have high parallel efficiency. In fact, in the case where a copy of the non-zero matrix elements of $A$ is sent to each processor, the execution time for computing an extremal eigenvalue on $p$ processors is bounded by $O(l N / p)$ where $N$ is the number of performed random walks and $l$ is the mean value of the steps in a single walk. This result assumes that the initial communication cost of distributing the matrix, and the final communication cost of collecting and averaging the distributed statistics is negligible compared to the cost of generating the Markov chains and forming the chosen statistic.

The numerical tests presented include example with $128 \times 128$ matrix, where we have applied rough Monte Carlo estimate and no refinement. In this case, the procedure for finding the largest eigenvalue of the inverse matrix using power Monte Carlo iterations, has no convergence. After that we applied a refinement procedure to find more accurate inverses with $\epsilon=10^{-6}$ and $10^{-8}$. Applying power Monte Carlo iterations to find the largest eigenvalue of the refined inverse matrix gives the value $\lambda I n v_{\max }=3.3330$ which is closed enough to the value given by the MATLAB eigenvalue procedure which is 3.3062 . 
In addition to convergence test, we also performed parallel computations to empirically confirm the parallel efficiency of this Monte Carlo method. A lot of parallel tests concerning Monte Carlo inversion and refinement can be found in [1], here we test the Monte Carlo part for computing the desired eigenvalue. The parallel numerical test was performed on a Compaq Alpha parallel cluster with 8 DS10 processors each running at 466 megahertz using MPI to provide the parallel calls. Each processor executes the same program for $N / p$ trajectories (here $p$ is the number of processors), and at the end of the trajectory computations, a designated host processor collects the results of all realizations and computes the desired average values. The results shown in Figure that the high parallel efficiency of Monte Carlo methods is preserved for this problem as well.

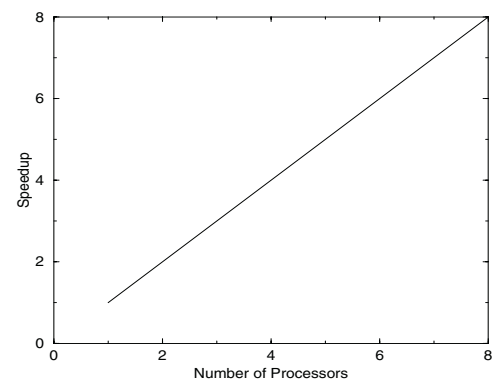

Fig. 1. Computing the largest eigenvalue of the inverse matrix - Speedup vs number of processors

\section{Conclusion}

The Monte Carlo methods with refinement have been proposed and extensively testes for solving systems of linear algebraic equations (see, for example, [1, 5]). In this paper we propose a method that includes Monte Carlo procedure for finding the inverse matrix, refinement procedure to improve approximation of the inverse if necessary, and Monte Carlo power iterations to compute the smallest eigenvalue. This method gives better results than the Resolvent Monte Carlo.

\section{References}

1. V. N. Alexandrov, S.Branford, and C. Weihrauch, A New Parallel Hybrid Monte Carlo Algorithm for Matrix Computations', Proc. Of PMAA 2004, September 2004.

2. I. Dimov, V. Alexandrov, A. Karaivanova, Resolvent Monte Carlo Methods for Linear Algebra Problems, Mathematics and Computers in Simulations, Vol. . 55, 2001, pp. 25-36. 
3. I. Dimov, T. Dimov, T. Gurov, A new iterative Monte Carlo Approach for Inverse Matrix Problem, J. of Comp. and Appl. Mathematics, 92, 1998, pp. 15-35.

4. I. Dimov, A. Karaivanova, Parallel computations of eigenvalues based on a Monte Carlo approach, Journal of MC Methods and Appl., Vol.4,Num.1, 1998 pp.33-52.

5. B. Fathi, B. Liu and V. Alexandrov, Mixed Monte Carlo Parallel Algorithms for Matrix Computations, Computational Science - ICCS2002, LNCS 2330, pp. 609618, 2002.

6. G. H. Golub, C.F. Van Loon, Matrix computations, The Johns Hopkins Univ. Press, Baltimore, 1996.

7. J.H. Halton, Sequential Monte Carlo Techniques for the Solution of Linear Systems, SIAM Journal of Scientific Computing, Vol.9, pp. 213-257, 1994.

8. William W. Hager, Applied Numerical Linear Algebra, Prentice Hall International (UK) Limited, London, 1989.

9. J.M. Hammersley, D.C. Handscomb, Monte Carlo methods, John Wiley \& Sons, inc., New York, London, Sydney, Methuen, 1964.

10. G.A. Mikhailov, Optimization of the "weight" Monte Carlo methods, Nauka, Moscow, 1987.

11. I.M. Sobol Monte Carlo numerical methods, Nauka, Moscow, 1973. 